\title{
The Biophysical Modeling of Gastric Circulation in The Human Organism
}

\author{
Janos Vincze* and Gabriella VinczeTiszay \\ Health Human International Environment Foundation, Budapest, Hungary \\ *Corresponding author: Janos Vincze,Health Human International Environment Foundation, Budapest, Hungary
}

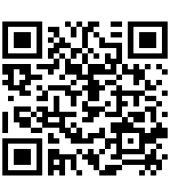

\section{ARTICLE INFO}

Received: 蔧 August 22, 2020

Published: 幽 September 08, 2020

Citation: Janos Vincze, Gabriella VinczeTiszay. The Biophysical Modeling of Gastric Circulation in The Human Organism. Biomed J Sci \& Tech Res 30(1)-2020. BJSTR. MS.ID.004909.

Keywords: Biophysical Modeling;Gastric Circulation;Rhythmic Model;Hypothetical Secundar Brain;Calorimetric Model;Adjustment

\begin{abstract}
Statistics from Hungary over the last seven years clearly show that diseases of the circulatory device lead the statistics on the causes of death. Consider the contraction and relaxation of the atrium/ventricle of the human heart; use the function of the rhythmic change for this. We apply the calorimetric principles for the measurement of the gastric blood flow. This way the test made can serve for the simultaneous measuring of the blood flow, the secretion and mobility of the stomach, before and after the administration of substances. In the dT time, the stomach wall passed an amount thermic energy to the balloon from the blood which passed through this portion in that period of time. The heating of the balloon is directly proportional with the amount of heat absorbed and inversely proportional with the balloon's thermal capacity. The human organism is a system because it is made up of a finite number of interacting $p_{1}, p_{2}, \ldots p_{n}$ elements, characterized by the quantitative degree of $\mathrm{q}_{1}, \mathrm{q}_{2}, \ldots \mathrm{q}_{\mathrm{n}}$. The circulatory apparatus is a subsystem of the human body. In our opinion, the circulatory device should have a control associated with its own structure, which is likely to consist of neurons with hyperordonated spatial structure, called the "hypothetical secondary brain", which performs certain control functions. This "hypothetical secondary brain" of the circulatory apparatus, in humans, functions continuously throughout their life
\end{abstract}

\section{Mini Review}

Now a days, most victims of humanity are the victims of cardiovascular diseases (Table 1). Only research in the relevant fields of biophysics, physiology, biochemistry, and pathophysiology can provide a solid basis for clarifying the cause, origin and effective prevention and cure of these diseases. Statistics from Hungary over the last seven years clearly show that diseases of the circulatory device lead the statistics on the causes of death. People with heart condition can expect the shortest life expectancy [1]. It cannot be emphasized enough that the stress caused by social and family environment contributes decisively to the development of heart disease and then to the death of the individual. The centuries-old folk proverb has been the result of a great deal of observation. It has been correctly recognized - without anatomical, biophysical, or physiological knowledge - that severe stress can lead to death. It is a well-known phenomenon that decades of happy marriage spent together is often followed by the rapid death of both parties. After a long-married life, it is noticeable that once the husband dies (the wife), within a few months the wife (the husband) dies, too. The
Hungarian trend is the same as the global trend, with circulatory system disease being the most common cause of death in every country.

Table 1: Hungarian statistical data on the causes of death.

\begin{tabular}{|c|c|c|c|c|c|c|}
\hline Year & Circul. & Tumour & Resp. & Digest. & Traffic & Total \\
\hline 2012 & 46.032 & 33.224 & 5088 & 3697 & 699 & 129.440 \\
\hline 2013 & 44.395 & 32.278 & 5292 & 3324 & 661 & 126.778 \\
\hline 2014 & 44.529 & 32.748 & 5212 & 3440 & 670 & 126.308 \\
\hline 2015 & 45.527 & 32.792 & 6266 & 3306 & 734 & 131.697 \\
\hline 2016 & 41.985 & 32.987 & 5490 & 3177 & 692 & 127.053 \\
\hline 2017 & 44.029 & 32.844 & 5110 & 3243 & 728 & 131.674 \\
\hline 2018 & 43.112 & 32.297 & 6093 & 3343 & 735 & 131.046 \\
\hline
\end{tabular}

\section{The Rhythmic Model of The Heart}

The circulatory device consists of the heart, which acts as the central pump, the blood vessels and lymphatic system that carry out transport. The heart consists of four compartments (two atria and 
two ventricles) in which the contraction (automation) is performed by the myocardium controlled on four levels. The four levels are: sinus-atrial node (70-80/min); atrial ventricular node (40 stimuli/ minute); the bundle of His (20-25/min) and the Purkinje network $(5-10 / \mathrm{min})$. The period which includes a single contraction (systole) and a single relaxation (diastole) is called cardiac cycle. The cardiac cycle lasts 0.8 seconds. Consider the contraction and relaxation of the atrium of the human heart [2]. Use the function of the rhythmic change for this. The atrial systole $\left(Z_{1}\right)$ is short as it lasts for only $0.1 \mathrm{~s}$, while the diastole $\left(\mathrm{Z}_{2}\right)$ is $0.7 \mathrm{~s}$ and the total period is $\mathrm{T}=0.8 \mathrm{~s}$. In this case, we only consider two states $\mathrm{k}=2$; $\mathrm{n}$ $\varepsilon(1,2, \ldots)$ - is the number of the cardiac cycle.

$$
F(t)= \begin{cases}Z_{1} & n .0 .8<t \leq n .0 .8+1 \\ Z_{2} & n .0 .8+1 \leq(n+1) 0.8\end{cases}
$$

Consider the contraction and relaxation of the ventricle of the human heart. Use the function of the rhythmic change for this. The ventricular systole $\left(\mathrm{Z}_{3}\right)$ lasts for $0.3 \mathrm{~s}$, while the diastole $\left(\mathrm{Z}_{4}\right)$ is 0.5 $\mathrm{s}$ and the total period is $\mathrm{T}=0.8 \mathrm{~s}$. In this case, we only consider two states $\mathrm{k}=2 ; \mathrm{n} \varepsilon(1,2, \ldots)-$ is the number of the cardiac cycle.

$$
F(t)= \begin{cases}Z_{3} & n .0 .8<t \leq n .0 .8+3 \\ Z_{4} & n .0 .8+3 \leq(n+1) 0.8\end{cases}
$$

Consider the contraction and relaxation of the human heart Use the function of the rhythmic change for this. The systole $\left(\mathrm{Z}_{5}\right)$ lasts $0.4 \mathrm{~s}$, while the diastole $\left(\mathrm{Z}_{6}\right)$ lasts $0.4 \mathrm{~s}$, the total period $\mathrm{T}=$ $0.8 \mathrm{~s}$. In this case, we only consider two states $\mathrm{k}=2$; where $\mathrm{n}$ is the heart rate in all three cases.

$$
F(t)= \begin{cases}Z_{5} & n .0 .8<t \leq n .0 .8+4 \\ Z_{6} & n .0 .8+5 \leq(n+1) 0.8\end{cases}
$$

After this break the cycle starts again. The cardiac muscle is never tired unless it suffered a pathological process.

\section{The Calorimetric Model of the Gastric Circulation}

The clinical and hemodynamic study of the vascular diseases mentioned the participation of many of the circulatory territories (cerebral, coronary, renal) in these diseases. The spleen circulation is more accessible to the hemodynamic investigation means. Further on we present a method of exploration of the gastric circulation [3] To apply the calorimetric principles for the measurement of the gastric blood flow, we made a probe at whose end a $20 \mathrm{ml}$ rubber balloon was attached. Inside the probe we introduced three thin tubes; two of them end inside the balloon and one crosses the balloon exiting it. At the end of one of the internal probes a thermistor was mounted, which can be placed in contact with a galvanometer. The external probe on whose end a Clive was mounted serving at introducing in the stomach some pharmacological substances or for feeding. This way the test made can serve for the simultaneous measuring of the blood flow, the secretion and mobility of the stomach, before and after the administration of substances. The principle lying at the base of the determination of the blood flow is the one of the calorimeters between the water in the balloon, the tissues of the stomach's walls and the blood that irrigates these tissues. Exchanges of caloric energy take place: at the beginning of the determination, the cold water from the balloon cools the organ's walls; they will be reheated by the flowing blood.

Due to this heat transfer in the last analysis, the temperature of the balloon water will start to rise, and it tends to equalise the one of the organ's wall [4]. There are two thermal systems with various temperatures: stomach $(0)$ having the temperature $t_{0}$ and the balloon with the temperature $t_{B}$ An unknown amount of blood of a constant temperature passes through the stomach's capillary system. Thus, at the beginning the stomach temperature and the blood temperature $\left(\mathrm{t}_{\mathrm{s}}\right)$ are the same, but when the stomach gets in contact with the balloon, which has a lower temperature, then it tends to equalize the temperature value. In the dT interval, the stomach wall passed an amount $\mathrm{Q}_{\mathrm{OB}}$ to the balloon from the blood which passed through this portion in that period. The heating of the balloon is directly proportional with the amount of heat absorbed and inversely proportional with the balloon's thermal capacity:

$$
d t_{B}=\frac{Q_{O B}}{C_{B}}
$$

On the other side, $Q_{O B}$ is proportional with the temperature difference, with the time interval and with the thermal transmission coefficient. We can write a similar equation for the stomach as well. The two equations can be reduced to the following forms:

$$
\begin{gathered}
Q_{O B}=a\left(t_{o}-t_{B}\right) d T \\
d t_{B}=\frac{a\left(t_{o}-t_{B}\right) d T}{C_{B}}
\end{gathered}
$$

where: $\mathrm{C}_{\mathrm{S}}$ is the blood's thermal capacity which passes in a time unit through $1 \mathrm{~cm}^{2}$ of the stomach's surface.

$$
\begin{gathered}
\frac{d t_{o}}{d T}=\left(-\frac{a}{C_{o}}-\frac{C_{V}}{C_{B}}\right) t_{o}+\frac{a}{C_{o}} t_{B}+\frac{C_{v}}{C_{o}} t_{V} \\
\frac{d t_{B}}{d T}=\frac{a}{C_{S}} t_{o}-\frac{a}{C_{B}} t_{B}
\end{gathered}
$$

To solve the problem, we must find the solution of the nonhomogenous differential equation solutions first [5]. So, the solution of the non-homogenous system will be:

$$
t_{o}=t_{S}-d \cdot e^{-r_{1} \cdot t}+d \cdot e^{-r_{2} \cdot t}
$$




$$
t_{B}=t_{S}-d \cdot K_{1} \cdot e^{-r_{1} \cdot t}+d \cdot K_{2} \cdot e^{-r_{2} \cdot t}
$$

\section{The Biophysical Adjustment}

By adjustment we understand the process through which a size is continuously measured and, by comparison with another reference value, it is influenced in the meaning of an equalization [6]. This way, the regulated unit is based on two components: comparison and command. Adjustment, with reference to the human body aims, within the biologically optimal limits of the structure and functionality, to all the steps of organization: maintaining the homeostasis of the internal environment, coordinated and unitary functionality of all its components, the adaptation of functionality to the requirements imposed by the environment. The circulatory apparatus is a subsystem of the human body. According to system theory, this phenomenon can be called a hierarchical order. This hierarchical order is broken down into further levels because the circulatory apparatus also has further subsystems. The circulatory device has its own "motor" - the heart, and a transport system, the blood. The function of the heart and blood vessels is constantly adapted to the requirements of the whole body and the different organs. The blood supply of the body and organs varies according to their function. This highly complex process of adaptation is accomplished through neural and humoral mechanisms that act simultaneously and coherently on both the heart and the vascular system. Nerve regulation operates on the principle of negative feedback [7]

The feed-back or negative inverse connection functions "constantly" and its main role is, to maintain certain functional parameters of the body to a relatively constant value. This is about a relative stability since the adjustment involves oscillations around an abstract medium value with a tendency to minimize the deviation in respect of this mean value. Let us denote with $\mathbf{o}(\mathbf{t})$ the exit output and the mean value of the characteristic parameter on the system is $\mathbf{o}^{*}(\mathbf{t})$; after the adjustment, the values of the outputs obtained shall be denoted with

$$
o\left(t_{1}\right), o\left(t_{2}\right) o\left(t_{3}\right), \ldots, o\left(t_{n}\right)=o^{*}(t) ;
$$

if

$$
\mathrm{t}_{1}<\mathrm{t}_{2}<\mathrm{t}_{3}<\ldots<\mathrm{t}_{\mathrm{n}} .
$$

We talk about a negative inverse value, if the following two conditions are satisfied:

$$
\begin{gathered}
\left|0^{*}(\mathrm{t})-\mathrm{o}\left(\mathrm{t}_{1}\right)\right|>\left|0^{*}(\mathrm{t})-\mathrm{o}\left(\mathrm{t}_{2}\right)\right|>\left|\mathrm{o}^{*}(\mathrm{t})-\mathrm{o}\left(\mathrm{t}_{3}\right)\right|>\ldots\left|\mathrm{o}^{*}(\mathrm{t})-\mathrm{o}\left(\mathrm{t}_{\mathrm{n}}\right)\right| \\
\frac{d\left|o\left(t_{i}\right)-o^{*}(t)\right|}{d t} \leq 0
\end{gathered}
$$

In the living organisms, all the mechanisms for maintaining a constant composition and internal environment function based on negative feedback. Effects from receptor zones. Receptors are found in both the heart and the large vessels [8]. The most important of these are those located in the «strategically» important areas of the circulatory system («reflexogenic zones»). There may be pressure sensors and chemoreceptors. These receptors capture changes in blood pressure or chemical composition, and send afferent stimuli to the cerebellar centers, mobilizing certain neural and humoral mechanisms that attempt to correct the changes resulted and restore the disturbed balance. Nerve centers that regulate the function of the heart and blood vessels is located in the reticular formation of the cerebellum. In this area of the nervous system, two types of neurons have been detected: some mainly regulate the function of the heart - heart centers - others regulate the tone of blood vessels - blood vessel centers. The large overlap between the two types of neurons justifies the area being called the cardio-vasomotor center. Even if the central nervous system is severely damaged in the brain, the circulatory system still works.

Therefore, in our opinion, the circulatory device should have a control associated with its own structure, which is likely to consist of neurons with hyperordonated spatial structure, called the "hypothetical secondary brain", which performs certain control functions [9]. This "hypothetical secondary brain" of the circulatory apparatus, in humans, functions continuously throughout their life, only so poorly controlled that it has not yet been detected and discovered by scientific research in addition to the dominant role of the central nervous system.

For the circulatory device, we focused on normal conditions, we did not discuss many perinormal or pathological conditions, nor did we discuss the effects of different drugs [10-12]. So, heart transplantation was not a subject matter either.

\section{Conclusion}

Biophysical modeling is a method that can be used to study biological mechanisms - that is, it involves mathematical analysis and model systems [13]. The models serve as analogues of operation and regulation, based on information signals specific to the living organism, and thus monitor their mechanism of operation until different automatic regulations of the normal state and different, that is deregulated functions and complexity [14]. Biophysical modeling means applying well-known physical laws to processes taking place in the living system. Medical biophysics investigates the application of physical laws to humans in the psychobiological system. To make this research successful, it uses mathematical language to model certain living structural or functional phenomena. The task of medical biophysics is to produce a device that contributes to a more accurate medical diagnosis [15] and to the application of physical methods that increase the quality of surgery. 


\section{References}

1. Vincze J (2018) Medical Biophysics. NDP P, Budapest.

2. Vincze J (2017) Biophysics of the Circular Apparatus. (2 $2^{\text {nd }}$ Edn.). NDP P, Budapest.

3. Vincze J (2015) The Capital Chapter of the Biophysics. ( $5^{\text {th }}$ Edn.). NDP P, Budapest.

4. Vincze J (2007) Interdisciplinarity. NDP P, Budapest.

5. Vincze J (2018) Biomathematics. NDP P, Budapest.

6. Vincze J, Vincze Tiszay G (2020) The Biophysical Adjustment in the Human Organism. J Med Res Case Reports (2) 3: 1-7.

7. Vincze J, Vincze Tiszay G (2020) The Biophysical Modeling of the Different Regulation in the Human Organism. Int J Innov Stud Med Sci (4) 1: 1-5.

8. Alberelli MA, De Candia E (2014) Functional role of protease activated receptor in vascular biology. Vascul. Pharmacol 62: 72-81.

\section{ISSN: 2574-1241}

DOI: $10.26717 /$ BJSTR.2020.30.004898

Janos Vincze. Biomed J Sci \& Tech Res

(C) (P) This work is licensed under Creative

Submission Link: https://biomedres.us/submit-manuscript.php
9. Vincze J, Vincze Tiszay G (2019) The "hypothetical secondary brain". Medical Research Archives 7(7): 1-3.

10. Chapman JC, Hajjar KA (2015) Fibrinolysis and the control of blood coagulation. Blood Rev 29: 17.

11. Versteeg HH, Heemskerk JWM, Levi M (2013) New fundamentals in hemostatic. Physiol Rev 93: 327.

12. Hoffmann JIE, Kaplan S (2002) The incidece of congenital heart disease. J Am Coll Cardiol 39: 1890.

13. Vincze J (2020) Biophysics of the Human Apparatus. NDP P, Budapest.

14.Vincze J (2020) The Biophysical Modelling of the Apparatuses in the Human Organism. Lambert Academic Publishing, Berlin.

15. Paumgatner GM (2010) Biliary physiology and disease reflections of a physician-scientist. Hepatology 51: 1095

\begin{tabular}{ll}
\hline \\
RIOMEDICAL
\end{tabular}$\quad \begin{aligned} & \text { Assets of Publishing with us } \\
& \text { - Global archiving of articles }\end{aligned}$

